\title{
ON A SOLUTION TO IMPROVE THE OBJECT DETECTION ABILITY OF RADARS BY DYNAMIC POLARIZATION METHOD
}

\author{
Nguyen Quoc An* \\ Le Quy Don University \\ 100 Hoang Quoc Viet, Hanoi, Vietnam
}

Received 10 February 2006

\begin{abstract}
One of the most importan problems of modern radar is increasing object detection, object distinction ability. In the last years, the used traditional radar signal processing methods are seem to use up. For solving this question, specialists are interested in analyzing the fine structure of radar signal, and the first is polarization structure. The use of polarization information allows to raise the information ability of radar systems and provides high probability of radar objects detection, high contrast of small-scale man-made objects on the radar map and radar objects classification.

The article deals with the detection of radar objects by exploiting the information on polarization based on the scattering matrix (SM) of the object. A solution to improve the object detection ability of radars by dynamic polarization method (to modify the polarization of radiative wave) is introduced.
\end{abstract}

\section{INTRODUCTION}

The polarization of the scattering wave by the object differs from that of radiative wave. Infomation on the object to be detected is contained in the polarization properties of the scattering wave and is to be expressed through the SM of the object.

Fundamentally, the SM of the object can be used to detect the radar object by applying the polarization selection method [3, 4]:

$$
S=\left(\begin{array}{ll}
\dot{S}_{11} & \dot{S}_{12} \\
\dot{S}_{21} & \dot{S}_{22}
\end{array}\right)
$$

If $\mathrm{S}$ is an arbitrary SM, by choossing the corresponding basic polarization, it will be transformed into a diagonal form, e. g $[4,6]$ :

*Corresponding author e-mail: quocan2410@yahoo.com.vn 


$$
S_{\text {dia }}=\left(\begin{array}{cc}
\lambda_{1} & 0 \\
0 & \lambda_{2}
\end{array}\right),
$$

where $\lambda_{1}$ and $\lambda_{2}$ are eigenvalues of the SM of radar object.

For the polarization radar, the following quantities can be used as detection parameters [6]:

1) The real parts and the imaginary parts of SM elements:

$$
\begin{aligned}
& \operatorname{Re} S_{11}=\lambda_{1} \cos ^{2} \gamma \cos 2 \eta+\lambda_{2} \sin ^{2} \gamma \cos (4 \alpha+2 \eta), \\
& \operatorname{Im} S_{11}=\lambda_{1} \cos ^{2} \gamma \sin 2 \eta+\lambda_{2} \sin ^{2} \gamma \sin (4 \alpha+2 \eta), \\
& \operatorname{Re} S_{12}=\left(\lambda_{2}-\lambda_{1}\right) \cos 2 \alpha \sin \gamma \cos \gamma, \\
& \operatorname{Im} S_{12}=\left(\lambda_{2}+\lambda_{1}\right) \sin 2 \alpha \sin \gamma \cos \gamma, \\
& \operatorname{Re} S_{22}=\lambda_{2} \cos ^{2} \gamma \cos 2 \eta+\lambda_{1} \sin ^{2} \gamma \cos (4 \alpha+2 \eta), \\
& \operatorname{Im} S_{22}=-\lambda_{2} \cos ^{2} \gamma \sin 2 \eta-\lambda_{1} \sin ^{2} \gamma \sin (4 \alpha+2 \eta) .
\end{aligned}
$$

2) The phases of SM elements:

$$
\psi_{j l}=\operatorname{arctg}\left(\frac{\operatorname{Im} S_{j l}}{\operatorname{Re} S_{j l}}\right) .
$$

3) The combinations of phases:

$$
\psi_{22}-\psi_{11} ; \quad \psi_{11}+\psi_{22}-2 \psi_{12} .
$$

4) The modules of the elements of SM and their squares:

$$
\begin{aligned}
& \left|S_{j l}\right|=\sqrt{\left(\operatorname{Re} S_{j l}\right)^{2}+\left(\operatorname{Im} S_{j l}\right)^{2}}, \\
& \left|S_{j l}\right|^{2} .
\end{aligned}
$$

5) The effective radar cross section (RCS) of the object:

$$
\sigma_{\Sigma}^{2}=\lambda_{1}^{2}+\lambda_{2}^{2} .
$$

6) The invariant properties of SM:

a) The determinant of SM:

$$
\operatorname{det} S=\lambda_{1} \lambda_{2}
$$

b) The polarization anisotropy coefficient of the object:

$$
q=\left|\frac{\lambda_{1}^{2}-\lambda_{2}^{2}}{\lambda_{1}^{2}+\lambda_{2}^{2}}\right| .
$$

In general, for fluctuation objects, $\lambda_{1}, \lambda_{2}, \eta, \gamma$ and $\alpha$ are random quantities. The obtained formulas have the characteristic that they are all expressed by a set of parameters $\lambda_{1}, \lambda_{2}, \eta, \gamma$ and $\alpha$ and are geometrically expressed by polarization ellipse and Poincaré sphere (unit sphere) [6].

Obviously, different parameters will provide different probabilities of detection $P_{D}[2,5$, 7]. For the same object and at the same moment, a change in the polarization of the radiative wave will 
lead to the change of the probability density function (pdf) or the variance of each element $S_{j l}$ of $\mathrm{SM}$; this change in turn leads to the change of the pdf of the combination of SM elements and accordingly to the change of $\mathrm{P}_{\mathrm{D}}$ with the same probability of false alarm as required. Therefore, the issue which element or combination of SM elements will be choosen as detection parameter would depend on the way to calculate the value of corresponding $\mathrm{P}_{\mathrm{D}}$ for $\mathrm{P}_{\text {Dmaxmax }}$ with the same required $\mathrm{P}_{\mathrm{F}}$. For this reason, this characteristic may be used to realize the method of radar object detection with highest probability of detection.

\section{A SOLUTION TO IMPROVE THE OBJECT DETECTION ABILITY OF RADARS BY DYNAMIC POLARIZATION METHOD}

In the condition of modified polarization level of radiative wave, we obtain the pdf of the modules of SM elements of ground clutter and ground clutter + object as presented in the table 1.

Table 1:

\begin{tabular}{lccc}
\hline Probability distributed law & Gaussian $\left(\mathbf{W}_{\mathbf{1}}\right)$ & Exponential $\left(\mathbf{W}_{\mathbf{2}}\right)$ & Rayleigh $\left(\mathbf{W}_{\mathbf{3}}\right)$ \\
\hline Gaussian $\left(\mathrm{W}_{1}\right)$ & $\mathrm{P}_{11}$ & $\mathrm{P}_{12}$ & $\mathrm{P}_{13}$ \\
Exponential $\left(\mathrm{W}_{2}\right)$ & $\mathrm{P}_{21}$ & $\mathrm{P}_{22}$ & $\mathrm{P}_{23}$ \\
Rayleigh $\left(\mathrm{W}_{3}\right)$ & $\mathrm{P}_{31}$ & $\mathrm{P}_{32}$ & $\mathrm{P}_{33}$ \\
\hline
\end{tabular}

In this table, $W_{i}(\sigma, x), i=\overline{1,3}$ are pdfs of ground clutter and ground clutter + object. $P_{k l}\left(\sigma_{1}, \sigma_{2}\right)(\mathrm{k}, \mathrm{l}=\overline{1,3})$ are probabilities of detection, $\sigma_{i}(\mathrm{i}=1,2)$ are variances of pdf. The probabilities of detection $P_{k l}$ are calculated according to Nayman - Pearson criteria $[1,8]$.

The problem of radar object detection in dynamic polarization mode with high probability of detection can be solved as follows. At an arbitrary state of polarization of order $\mathrm{j}$ $(j=1,2, \ldots, \mathrm{M}), \mathrm{P}_{\mathrm{Dj}}$ is successively calculated corresponding to each combination and then for $P_{D \max j}=\max \left\{P_{D j}\right\}$. After $\mathrm{M}$ times of changes of the polarization state of radiative wave, $\mathrm{P}_{\text {Dmaxmax }}$ is calculated as $P_{D \max \max }=\max \left\{P_{D \max j}\right\}(j=1,2, \ldots, \mathrm{M})$.

Hence, an element of SM or a combination of SM elements will be choosen as detection parameter for $\mathrm{P}_{\text {Dmaxmax }}$. The above procedure is used to calculate the maximum of multidirectional function $\mathrm{P}_{\mathrm{D}}$.

Figure 1 represents the probabilities of detection $P_{12}$ and $P_{23}$ where pdfs ground clutter and ground clutter + object are Gaussian - Exponential and Exponential - Rayleigh, respectively.

The comparison of probabilities of detection $P_{k l}$ giving polarization changes is shown in Fig. 2 . Their values are dependent on the pdf and the characteristics of pdf (expected value, variance, etc). It shows that, to detect the radar object the dynamic polarization method is preferred since it provides the highest probability of detection.

That is the reason why, we introduce in this work a block diagram of the system to improve the object detection ability of radars using dynamic polarization method (Fig. 3). 

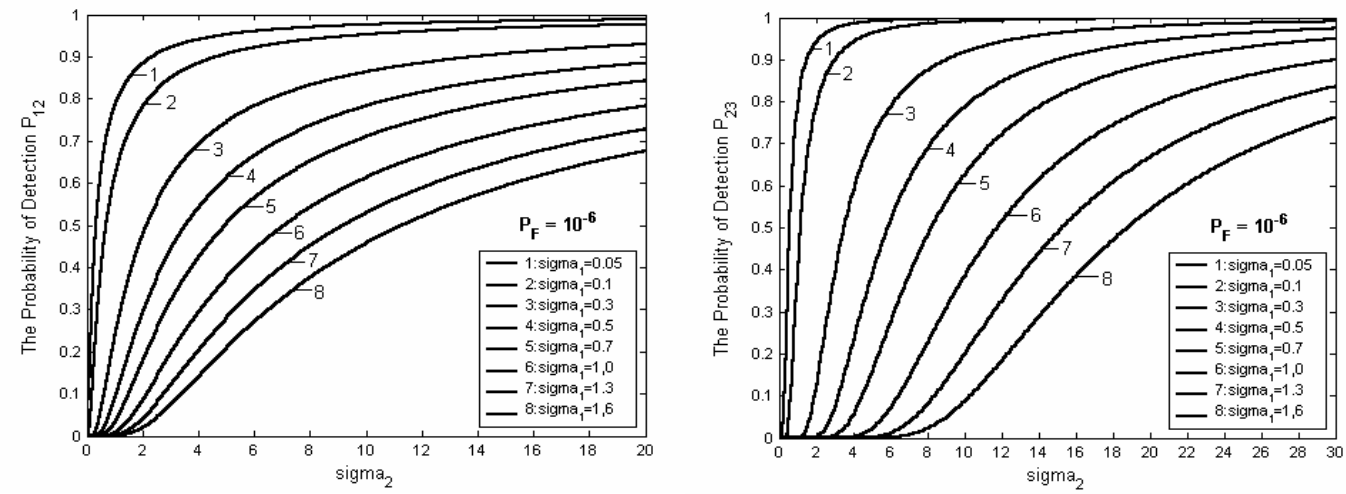

Fig. 1: $\quad$ The probabilities of detection $P_{12}$ and $P_{23}$ where pdfs ground clutter and ground clutter + object are Gaussian - Exponential and Exponential - Rayleigh, respectively
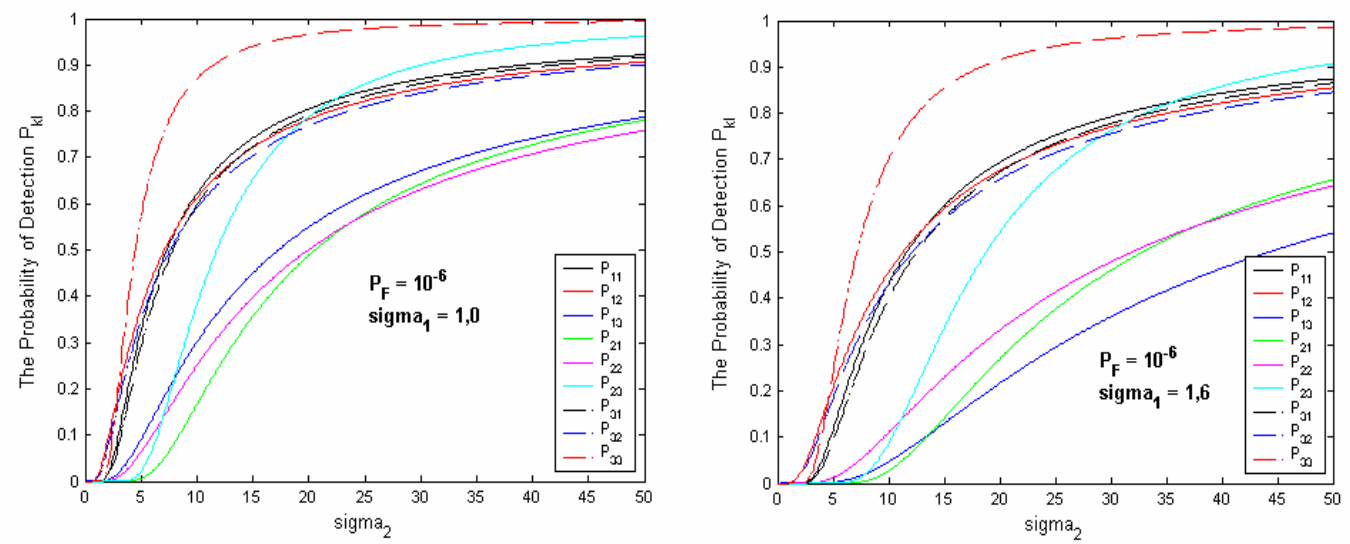

Fig. 2: $\quad$ The comparison of probabilities of detection $P_{k l}$ giving polarization changes

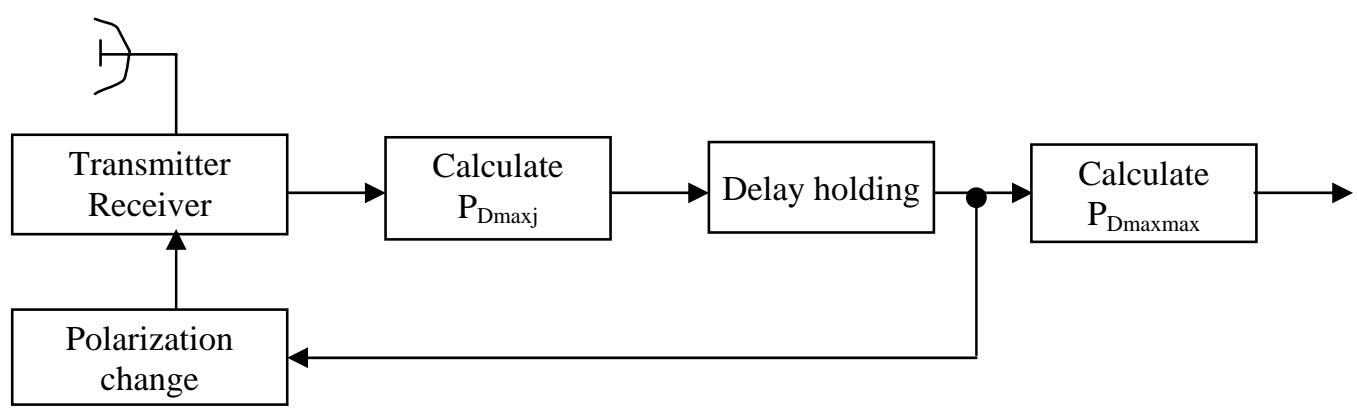

Fig. 3: The sketch of the system to improve the object detection ability of radars using dynamic 110 polarization method 
- Block "Polarization change" changes values of polarization parameters of radiative wave.

- Block "Calculate $P_{\text {Dmaxi" }}$ calculates successively the probability of detection corresponding to each SM elements and their combinations in each order $\mathrm{j}$ of polarization mode and calculating $\mathrm{P}_{\mathrm{Dmaxj}}$ corresponding to this polarization state.

- $\quad$ Block "Delay holding" stores all values of $\mathrm{P}_{\operatorname{Dmaxj}}(j=\overline{1, M})$ each time the polarization is changed.

- $\quad$ Block "Calculate $\mathrm{P}_{\mathrm{Dmax} \max }$ " finds the highest probability of detection after each period (M times) of polarization change; while, remembering the pair of parameters and the polarization state corresponding to $\mathrm{P}_{\mathrm{Dmaxmax}}$, the object will then be detected with the highest probability of detection.

When receiving reflex signal, the detector of radar will determine the probability distribution of SM elements and their combinations. Then, it will successively calculate the probability of detection corresponding to each pair of SM parameters of ground clutter and ground clutter + object, respectively. Based on the probabilities of detection, the detector will determine the highest probability of detection at each order $\mathrm{j}$ of polarization state, i. e $\mathrm{P}_{\text {Dmaxj }}$.

Therefore, at a certain moment, if a object is continuosly illuminated by a polarization radiative wave, the detector of radar will determine the highest probability of detection $P_{D \max \max }=\max \left\{P_{D \max j}\right\}(j=\overline{1, M})$. At the same time, the pair of parameters of SM corresponding to $\mathrm{P}_{\mathrm{Dmax} \max }$ will be choosen as detection parameters. The meaning of the problem in this case is to calculate the maximum values of multi-directional functions $P_{D}$ in the considered changing domain.
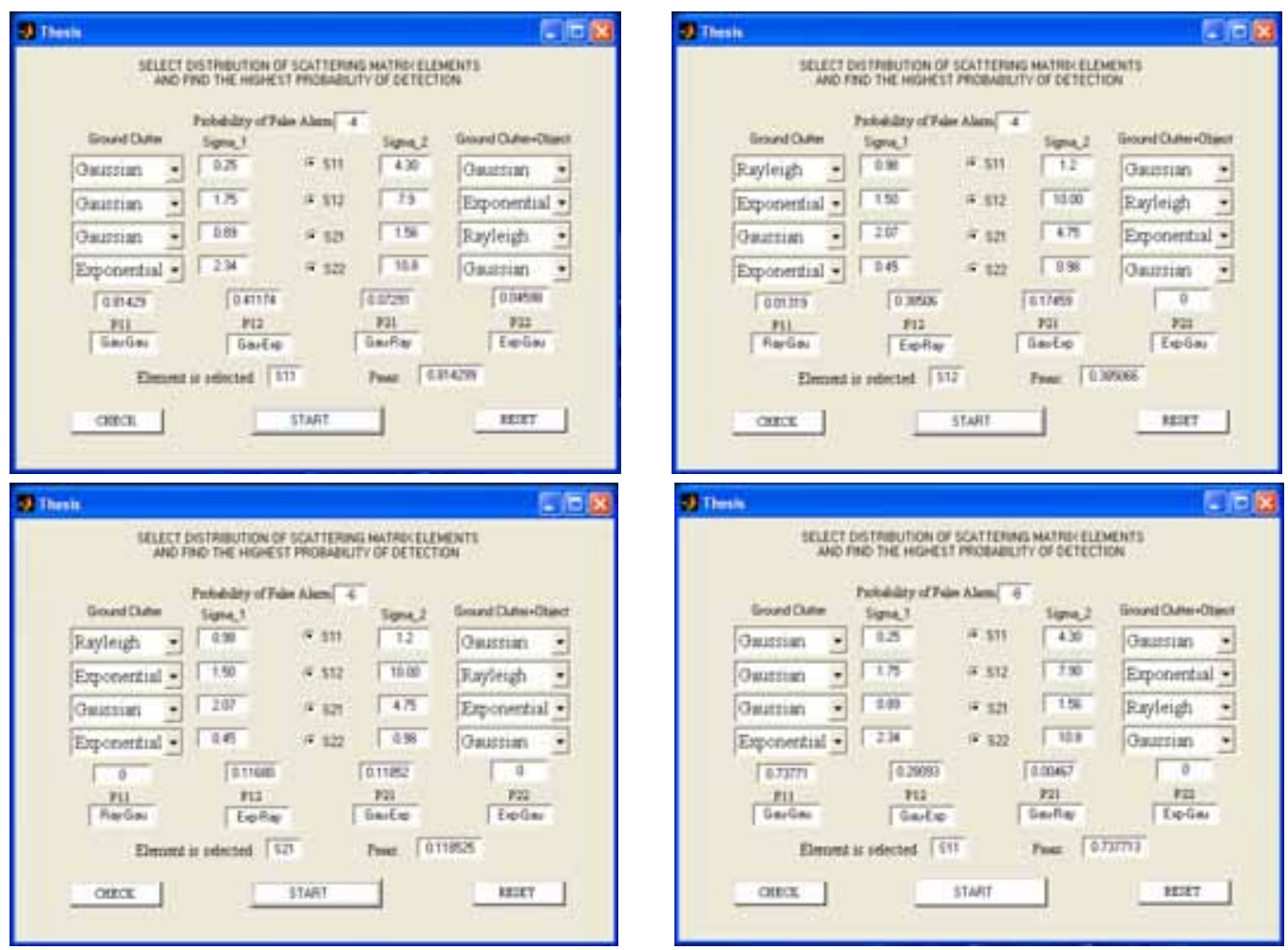

Fig. 4: $\quad$ Interfaces, describing the algorithm of selection of detection parameters and the calculation of the highest probability of detection 
The algorithm, describing the selection of detection parameters and the calculation of the highest probability of detection is as follows:

- Choose each element $\dot{S}_{i j}$ of the SM of ground clutter and ground clutter + object;

- Determine the variances $\sigma_{1}$ and $\sigma_{2}$ for ground clutter and ground clutter + object;

- Determine the distribution $W_{j}(\sigma, x)(j=1,2,3)$ for element $\dot{S}_{i j}$;

- Based on values of $\dot{S}_{i j}, \sigma_{1}, \sigma_{2}$ and $\mathrm{W}_{\mathrm{j}}$, calculate values of $\mathrm{P}_{\mathrm{kl}}$, and then determine $P_{D \max j}=\max \left\{P_{k l}\right\}$.

- The interface and obtained values are showed in Fig. 4. We assume that, pdf of SM elements of ground clutter and ground clutter + object after $\mathrm{M}$ times of change of polarization state are Gaussian, Exponential and Rayleigh as indicated in the Table 1.

\section{CONCLUSIONS}

In this paper we show that, the distribution or variance of SM elements will be changed when the polarization of radiative wave is modified. This change will lead to the change in the probability of detection of radar objects. In the dynamic polarization mode (where the polarization of radiative wave is modified), the radar object will be detected with the highest probability of detection corresponding to a given probability of false alarm.

\section{REFERENCES}

1. Nguyen Duc Luyen (2003), Radar principles, Military Technical University, vol. 213 (in Vietnamese).

2. Dao Chi Thanh (2002), On a solution of fast radar target detection for radar stations radiate waes have changed polarization in the automatic air traffic control system, Proceeding of science conference, Institute of Mechanics, Vietnamese Academy of Science and Technology, pp. 150-157 (in Vietnamese).

3. Kanarejkin, D.B., Pavlov, N.F., and Potechin, V.A. (1966), Radar Signal Polarization, M.: Radio and Sviaz, 440 (in Russian).

4. Bogorodsky, V.V., Kanarejkin, D.B., and Kozlov, A.I. (1981), Polarization of scattered and own radion emission of terrestrial covers, L.: Gidrometeoizdat, 280 (in Russian).

5. Dao Chi Thanh (2001), A method using dynamic polarization mode for improving the difference of radar targets, Bulletine of Moscow State Technical University of Civil Aviation (MSTUCA), Ser. Radiophysics and Radiotechnics, vol. 24, pp. 233-234 (in Russian).

6. Saratov, V.A. (1995), Statistical model of polarization characteristics of radar targets, A dissertation submited to the MSTUCA for the degree of Doctor of Philosophy, 205 (in Russian).

7. Dao Chi Thanh and Nguyen Quoc An (2004), A solution for fast radar target detection with high detection probability using dynamic polarization method, The VII International Conference: The actual problem of electronical devices design and manufacturing, session "Polarization radar", 21-24 September 2004, Novosibirsk, Russia.

8. Bassem, R. and Mahafza, Ph.D. (2000), Radar Systems Analysis and Design Using MATLAB, Pressed by Chapman \& Hall/CRC. 
AJSTD Vol. 23 Issues 1\&2

9. Adrian Brian and Moshe Breiner (1996), MALAB for Engineers, University Press, Cambridge. 